\title{
Potency of Dry Needling As A Pain Modulating Modality in Shoulder Pains
}

\author{
Dr. N. Ravinder Kumar ${ }^{1,}$ Dr. Kodanda Pani ${ }^{2}$ Dr. Kali Vara Prasad Vadlamani ${ }^{3,}$ \\ Dr. G.V.S. Murthy ${ }^{4}$, Nirmala Kajjam ${ }^{5}$, Arthi Gavatre $^{6}$, Kusuma Shanti ${ }^{7}$, \\ Deepthi .G. ${ }^{8}$ \\ ( 1\&3 Associate Professors, 2\&4 Professors, Department Of Orthopedics \& Traumatology, Osmania Medical \\ College/ Hospital, 4,5,6 \&7 physiotherapy Department, Osmania Medical College, Hyderabad).
}

Background: Myofascial Pain Syndrome (MPS) is a significant health problem affecting as much as $85 \%$ of the general population, sometime in their lifetime, while the estimated overall prevalence is $46 \%$. Shoulder pain is the third most common MPS affecting all age groups with no gender discrimination. It can be caused by a variety of disorders like periarthritis, impingement syndromes, degeneration and trauma. Certain medical conditions like diabetes can precipitate shoulder pain and some conditions like gallbladder disorders liver and heat problems can cause pain referred to the shoulder. Self-reported prevalence of shoulder pain is between $16 \%$ and 26\%. Trigger point dry needling or simply dry needling does intramuscular stimulation and is a skilled invasive intervention that uses a " dry' needle, one without medication or injection ,inserted through the skin into areas of the muscle, stimulate underlying myofascial trigger points, muscular and connective tissues for the management of neuromusculoskeletal pain and movement impairments in Myofascial Pain Syndrome (MPS).

Materials\& Methods: 76 patients (45 male and 31 female) who attended the Department of PhysioOccupational Therapy, which is a part of the Department of Orthopaedics, Osmania General Hospital, were randomly chosen after clearance from the Ethical Committee. The study period extended from June 2015 to June 2016. They were divided into groups according to their position in Visual Analogue Scale (VAS) and their respective scores were noted. Their scores were once again noted after they received the Dry Needling treatment of about 6 sittings.

Results: Out of the 77 cases, 57 cases showed excellent results (VAS 0-1 after treatment), 14 cases showed good results (VAS 2-3 after treatment), 5 cases showed fair results (VAS 4-5 after treatment),

Conclusion: Dry Needling is a relatively new treatment modality with specific subjective pain modulation efficacy in myofascial pain syndrome, which can help us in alleviating the pain in chronic conditions and acts adjuvant to the specific treatment.

Keywords: Dry Needling, Myofascial Pain Syndrome (MPS), Myofascial Trigger Points, VAS.

\section{Introduction}

Myofascial pain syndrome (MPS) is a fancy way to describe muscle pain. It refers to pain and inflammation in the body's soft tissues. MPS is a chronic condition that affects the fascia (connective tissue that covers the muscles). It may involve either a single muscle or a muscle group. In some cases, the area where a person experiences the pain may not be where the myofascial pain generator is located. The actual site of the injury or the strain prompts the development of a trigger point that, in turn, causes pain in other areas. This situation is known as referred pain.

Trigger points are physiological contractures characterised by local ischemia, hypoxia, significantly lower $\mathrm{pH}$, altered chemical milieu, local and referred pain and altered muscle activation pattern. This area becomes painful at the site and can also "radiate" in predictable patterns known as referred pain. Trigger points (TP) may be of two types - Active and Latent. An active TP exhibits spontaneous pain or pain in response to movement, stretch or compression, while a latent TP is a sensitive spot with pain or discomfort in response to compression only. Yet treatment of active TPs may be necessary in situations in which active TPs persist even after the underlying aetiologic lesion has been appropriately treated. ${ }^{1}$

Dry Needling is a invasive procedure, in which a solid filiform needle (Also referred to as acupuncture needles) or hollow-core hypodermic needle is inserted into the skin and muscle directly at a trigger point for alleviation of chronic muscle pain including pain related to myofascial pain syndrome through Intramuscular Stimulation (IMS). Dry Needling causes a Local Twitch Response (LTR) by stimulating the concerned muscle. LTR is spinal cord reflex that is characterised by a momentary involuntary contraction of the muscle. This leads to relaxation of shortened taut bands of muscle fibres, which can be elicited by snapping palpation. Eliciting this 
local twitch response is a key for successful deactivation of the trigger point, which releases muscle tension and lessens the pain to bring immediate relief from symptoms.

\section{Dry needling modulates subjective pain sensation through the following effects:}

Effects on the Taut Band Insertion of a needle at the endplate region- may lead to increased discharges leading to elicitation of LTRs and reduction of available acetylcholine stores. LTR causes alterations in the length and tension of the muscle fibres and stimulates mechanoreceptors like the $\mathrm{A} \beta$-fibres. ${ }^{2}$

Effects on Blood Flow - Dry Needling may increase muscle blood flow and oxygenation countering the local ischemia and hypoxia in the core of the TPs caused by sustained contractures of taut muscle bands. Probably, the release of vasoactive substance such as CGRP (calcitonin gene related peptide) and Substance P (SP) which upon activation of $\mathrm{A} \delta$ - and $\mathrm{C}$-fibres via the axon reflex lead to vasodilatation of small vessels, thus increasing the blood flow. ${ }^{3}$

\section{Neurophysiological effects}

\section{Effects on peripheral sensitization-}

Shah et al4,5 found that the concentrations of SP and CGRP were higher in the vicinity of active TPs compared to latent ones or normal muscle tissue. ${ }^{4.5}$ After a LTR was elicited, SP and CGRP concentrations were significantly lowered compared to their pre-LTR values. These values were consistent with the data of Hsieh et al. ${ }^{6}$ A single session treatment produced a short-term analgesic effect by decreasing the SP at peripheral sites.

Effects on Central Sensitization-

The most likely mechanism of pain relief through needle stimulation is hyper stimulation analgesia. DN probably stimulates both large myelinated fibers (A $\beta$ - and $A \delta$-fibers) as well as $C$-fibers indirectly via the release of inflammatory mediators. As a result of mechanical stimulation, $\mathrm{A} \beta$ - and $\mathrm{A} \delta$ - fibers are both activated and send afferent signals to the dorsolateral tracts of the spinal cord and activate the supraspinal and higher centres involved in pain processing. ${ }^{7,8,9,10}$ The advantages of Dry Needling are many. Quicker results, no drugs, symptomatic relief, cost effectiveness, treatment of deeper layers of muscle, parts of the muscle all make it an excellent pain modulating procedure. Our study was an attempt at establishing the pain modulating efficacy of Dry Needling, as it is simple yet effective technique for providing symptomatic relief from chronic muscular pain.

\section{Materials And Methods}

76 patients ( 45 male and 31 female, age range from 20 years to 60 years) who attended the Department of Physio- Occupation Therapy, which is a part of the Department of Orthopaedics, Osmania General Hospital were randomly chosen. The study period extended from June 2015 to June 2016, a minimum of 4-6 sittings were given.

\section{Inclusion Criteria}

Patients between the ages of 20-70 yrs.

Patients whose pain was not relieved with traditional physiotherapy methods.

Patients with VAS score 5 and above.

\section{Exclusion Criteria}

Patients with Paraesthesia, anxiety, renal failure, cardiac failure, uncontrolled diabetes, vascular diseases like haemophilia or on blood thinning medications.

A comparative Visual Analogue Scale (VAS) was used to divide the subjects into 6 groups according to their position in the scale. The patients were so selected that they had chronic pain in the muscles of the back and were placed after position 5 in the scale. Moreover, all of them were not getting pain relief with traditional mode of treatment. Dry Needling treatment was given after identifying the position of major trigger points of the back (Diagram 1,2). A VAS is a simple and frequently used method for the assessment of variations in intensity of pain. In clinical practice the percentage of pain relief assessed by VAS is often considered as a measure of the efficacy of treatment. Operationally VAS is a pain rating scale anchored by word descriptors at one side and facial illustration on the other side. ${ }^{11}$ (Diagram 3).

\section{Results}

Out of the 76 cases, 57 cases showed excellent results (VAS 0- 1 after treatment), 14 cases showed good results (VAS 2-3 after treatment), 5 cases showed fair results (VAS 4-5 after treatment), (Table 1, graphs 1 showing cumulative results graphs 2 to 6 showing individual results ) 


\section{Discussion}

The shoulder is the most movable joint in the human body. A group of 4 muscles and their tendons, called the rotator cuff, give the shoulder its wide range of motion

Most shoulder problems fall into four major categories:

- Tendon inflammation (bursitis or tendinitis) or tendon tear

- Instability

- Degenerative

- Traumatic

- Impingements

Other much less common causes of shoulder pain are tumours, infection, and nerve-related problems. The most common upper body primary myofascial syndrome is Periarthritis, involving the subscapularis, supraspinatus, infraspinatus and teres minor muscles. The other muscles involved in shoulder pain are the deltoid, lattissimus dorsi , trapezius and pectoral muscles. The rhomboids muscle also has major role in shoulder pains. Rhomboid trigger point pain is typically masked by the pain from trigger points in the levator scapulae, trapezius, and infraspinatus muscles. It usually becomes apparent only after these other trigger points have been released. Another interesting fact is the scalene muscle also can show referred pain in the shoulder, arm, neck, chest and upper back.

\section{Conclusion}

Dry Needling is a modality to facilitate a quick response to significantly reduce pain, so a person could return to function. It is minimally invasive, cheap, carries low risk, and reduces medication. It is an excellent adjuvant therapy in the management of chronic painful conditions like shoulder pains and other forms of myofascial pains.

\section{References}

[1]. Hong CZ. Treatment of myofascial pain syndrome. Curr Pain Headache Rep 2006;10(5):345-9. 2iagram 2: Dry Needling P

[2]. Baldry P. Acupuncture, trigger points and musculoskeletal pain, 3rd ed. Churchill Livingstone 2005.

[3]. Cagnie B, Barbe T, De Ridder E, et al. The influence of dry needling of the trapezius muscle on muscle blood flow and oxygenation. J Manipulative Physiol Ther 2012;35(9):685-91.

[4]. Shah JP, Phillips T, Danoff JV, et al. An in vivo microanalytical technique for measuring the local biochemical milieu of human skeletal muscle. J Appl Physiol 2005;99(5):1977-84.

[5]. Shah JP, Gilliams EA. Uncovering the biochemical milieu of myofascial trigger points using in vivo microdialysis: an application of muscle pain concepts to myofascial pain syndrome. J Bodyw Mov Ther 2008;12(4):371-84.

[6]. Hsieh YL, Yang SA, Yang CC, et al. Dry needling at myofascial trigger spots of rabbit skeletal muscles modulates the biochemicals associated with pain, inflammation, and hypoxia. Evid Based Complement Alternat Med 2012;2012:342165.

[7]. Chou LW, Kao MJ, Lin JG. Probable mechanisms of needling therapies for myofascial pain control. Evid Based Complement Alternat Med 2012;2012:705327. 8. Chu J, Schwartz I. The muscle twitch in myofascial pain relief: effects of acupuncture and other needling methods. Electromyogr Clin Neurophysiol 2002;42(5):307-11.

[8]. Chu J, Schwartz I. The muscle twitch in myofascial pain relief: effects of acupuncture and other needling methods. Electromyogr Clin Neurophysiol 2002;42(5):307-11.

[9]. Srbely JZ, Dickey JP, Lee D, et al. Dry needle stimulation of myofascial trigger points evokes segmental anti- nociceptive effects. J Rehabil Med 2010;42(5):463-8

[10]. Hsieh YL, Kao MJ, Kuan TS, et al. Dry needling to a key myofascial trigger point may reduce the irritability of satellite MTrPs. Am J Phys Med Rehabil 2007;86(5):397- 403.

[11]. Visual analog scale and verbal pain intensity scale. From pain management: theory and practice, edited by RK Portenoy, RM Tanner, copyright by Oxford University Press, Inc. Used by permission of Oxford University.

Table 1: Results - VAS scores before and after Treatment.

\begin{tabular}{|l|l|l|l|l|}
\hline $\begin{array}{l}\text { No. } \\
\text { of } \\
\text { Cases }\end{array}$ & & $\begin{array}{l}\text { VAS } \\
\text { before } \\
\text { Treatment }\end{array}$ & $\begin{array}{l}\text { No. of } \\
\text { Cases }\end{array}$ & $\begin{array}{l}\text { VAS after } \\
\text { Treatment }\end{array}$ \\
\hline 2 & \multirow{2}{*}{} & 10 & 1 & 0 \\
\hline 11 & \multirow{2}{*}{} & \multirow{2}{*}{} & 1 & 3 \\
\hline & \multirow{2}{*}{} & 3 & 0 \\
\hline & & 1 & 2 \\
\hline
\end{tabular}


Potency Of Dry Needling As A Pain Modulating Modality In Shoulder Pains

\begin{tabular}{|c|c|c|c|}
\hline & & 2 & 4 \\
\hline \multirow[t]{6}{*}{40} & \multirow[t]{6}{*}{8} & 14 & 0 \\
\hline & & 16 & 1 \\
\hline & & 6 & 2 \\
\hline & & 2 & 3 \\
\hline & & 1 & 4 \\
\hline & & 1 & 5 \\
\hline \multirow[t]{4}{*}{13} & \multirow[t]{4}{*}{7} & 7 & 0 \\
\hline & & 3 & 1 \\
\hline & & 2 & 3 \\
\hline & & 1 & 4 \\
\hline \multirow[t]{3}{*}{9} & \multirow[t]{3}{*}{6} & 5 & 0 \\
\hline & & 3 & 1 \\
\hline & & 1 & 3 \\
\hline 1 & 5 & 0 & 1 \\
\hline
\end{tabular}

